\title{
BMJ Open Hypertensive disorders of pregnancy and risk of asthma in offspring: protocol for a systematic review and meta- analysis
}

Ping Li, ${ }^{1,2}$ Tao Xiong (D) , ${ }^{1,2,3}$ Yong $\mathrm{Hu}^{1,2}$

To cite: Li P, Xiong T, Hu Y. Hypertensive disorders of pregnancy and risk of asthma in offspring: protocol for a systematic review and meta-analysis. BMJ Open 2020;10:e035145. doi:10.1136/ bmjopen-2019-035145

- Prepublication history and additional material for this paper are available online. To view these files, please visit the journal online (http://dx.doi. org/10.1136/bmjopen-2019035145).

Received 20 0ctober 2019 Revised 21 March 2020 Accepted 23 March 2020

Check for updates

(c) Author(s) (or their employer(s)) 2020. Re-use permitted under CC BY-NC. No commercial re-use. See rights and permissions. Published by BMJ.

'Department of Pediatrics, West China Second University Hospital, Sichuan University, Chengdu, Sichuan, China ${ }^{2}$ Key Laboratory of Birth Defects and Related Diseases of Women and Children (Sichuan University) Ministry of Education, Chengdu, China

${ }^{3}$ Deep Underground Space Medical Center, West China Hospital, Sichuan University, Chengdu, China

Correspondence to

Dr Tao Xiong;

tao_xiong@126.com

\section{ABSTRACT}

Introduction Hypertensive disorders of pregnancy (HDP), one of the most common obstetrical complications, has been reported to have a controversial relationship with the increased risk of asthma in offspring. No systematic review of this topic has been performed. The aim of this systematic review will be to summarise the available evidence examining the association between HDP and the risk of asthma in offspring.

Methods and analysis We will follow the Preferred Reporting Items for Systematic Reviews and MetaAnalyses and Meta-analysis of Observational Studies in Epidemiology guidelines. A systematic search of the PubMed, Embase, Cochrane and Web of Science databases will be performed using a detailed search strategy from database inception through 31 December 2019. Cohort, case-control and cross-sectional studies that report a diagnosis of maternal HDP and asthma in offspring will be included. Studies will be limited to the English language and include only human participants. Two independent reviewers will conduct the study selection, data extraction and risk of bias assessments using a standardised data extraction form. A meta-analysis will be performed to calculate overall pooled estimates using the generic inverse variance method. The data will be synthesised by either fixed-effect or random effects models according to heterogeneity tests. All analyses will be performed in Stata 14 and RevMan 5.3. High-quality evidence of the relationship between HDP and the risk of asthma in exposed offspring will be identified through the synthesis of current studies. In addition, the results of subgroup analyses and related secondary outcomes will be reported. The following will be concluded: (i) whether HDP increases the risk of asthma in offspring, (ii) whether HDP affects the severity of asthma in exposed offspring and (iii) whether possible differences in the risk of asthma among different HDP subgroups exist.

Ethics and dissemination There is no requirement for ethics approval because the meta-analysis and systematic review will be based on published data. It is anticipated that the dissemination of results will take place at conferences and through publication in a peer-reviewed journal.

\section{INTRODUCTION}

Hypertensive disorders of pregnancy (HDP) is estimated to affect $5 \%$ to $15 \%$ of all pregnancies and is recognised as one of the most
Strengths and limitations of this study

- This will be the first meta-analysis to explore the relationship between hypertensive disorders of pregnancy and the risk of asthma in exposed offspring.

- This protocol was designed following the Preferred Reporting Items for Systematic Reviews and MetaAnalyses and Meta-analysis of Observational Studies in Epidemiology guidelines.

- Since there may be some potential confounding factors, we will perform detailed subgroup analyses to reduce the effects of confounding factors.

- Since positive results are more prone to be published online, funnel plots will be used to assess publication bias.

- Selection bias will be considered because the pooled data will be obtained from observational studies.

common obstetrical complications. ${ }^{1}{ }^{2}$ HDP includes any hypertensive condition before gestation or manifested before 20 weeks and hypertension starting at or after 20 weeks; types of hypertension include gestational hypertension $(\mathrm{GH})$, preeclampsia $(\mathrm{PE})$, chronic hypertension and PE superimposed on chronic hypertension according to the International Society for the Study of Hypertension in Pregnancy. ${ }^{3}$ Recent studies have reported that HDP is closely associated with an increased risk of diseases in offspring, including asthma, allergy, eczema, ${ }^{4}$ high blood pressure, ${ }^{5}$ congenital heart defects, ${ }^{6}$ obesity, ${ }^{7}$ autism spectrum disorder, attentiondeficit/hyperactivity disorder, low cognitive function, anxiety/depression and other neurodevelopmental disorders. ${ }^{89}$ The 'Developmental Origins of Health and Disease' hypothesis, which has been widely confirmed, suggests that fetuses exposed to adverse uterine environments are prone to develop chronic non-communicable diseases later in life owing to perpetual alterations in the fetal vasculature, cardiac structure, pancreas, 
adipose tissue and brain structure. ${ }^{10} 11$ In addition, HDP induces a detrimental in utero environment, with systemic inflammation and oxidative stress experienced by the fetus, thus impairing vulnerable fetal lung development and disturbing immune function, which may persist throughout life in the offspring.

Asthma, a common lung disease associated with abnormal inflammation and immune response, is characterised by variable symptoms of wheezing, shortness of breath, chest tightness and/or cough, and variable expiratory airflow limitation. It is a chronic disease of childhood and is responsible for the majority school absences, emergency department visits and hospitalisations. Thus, asthma has been regarded as a major public health challenge worldwide, affecting $1 \%$ to $18 \%$ of populations in different countries. ${ }^{12} 13$ The development of asthma has generally been attributed to the interactions between genetics and environmental factors. ${ }^{14}$ Currently, it is widely accepted that asthma develops early in life. ${ }^{16}$ Recurrent wheezing and other asthma-like symptoms usually begin within the first few weeks or months after birth. There is increasing evidence to suggest that negative events occurring early in life, even in the perinatal period, significantly increase the risk of asthma and poor lung function later in life. ${ }^{17} 18$ Hence, adverse obstetrical events, such as HDP, may have strong effects on fetal airway structure and lung and immune system development during the prenatal period and may significantly increase the susceptibility of offspring to asthma. ${ }^{19} 20$

Approximately half of individuals with asthma are reported to have suffered from asthma-like symptoms during childhood. ${ }^{21}$ Therefore, early recognition and intervention are critical to reduce short-term and longterm morbidity and to prevent potential long-term sequelae resulting in impaired lung function. Recent studies have shown that HDP is a potential risk factor for childhood asthma in offspring. ${ }^{422}$ However, the association between HDP and asthma is controversial. ${ }^{23}$ Identifying the relationship between HDP and asthma could help us to understand the pathogenesis of asthma. It also facilitates early asthma recognition and intervention in high-risk populations. Therefore, we aim to perform a systematic review and meta-analysis to summarise the available evidence regarding the relationship between HDP and asthma in offspring.

\section{OBJECTIVES}

The aim of the present systematic review and metaanalysis is to summarise the available evidence to explore the following:

1. Whether HDP increases the risk of asthma in offspring;

2. Whether HDP affects the severity of asthma in exposed offspring; and

3. Whether differences in the risk of asthma among different HDP subgroups exist.

The study will be conducted based on the following requirements.

\section{Population}

Pregnant women and their offspring at any age (both child offspring and adult offspring) will be included.

\section{Intervention/exposures}

HDP, defined as any hypertension (systolic blood pressure (BP) $\geq 140$ and/or diastolic $\mathrm{BP} \geq 90 \mathrm{~mm} \mathrm{Hg}$ ) during pregnancy, mainly includes the following:

1. GH: any hypertensive disorder that develops at or after 20 weeks of gestation in the absence of features of PE;

2. PE: GH accompanied by one or more of the following new-onset conditions at or after 20 weeks of gestation: (i) proteinuria, (ii) maternal organ dysfunction, such as acute kidney injury, liver complications, neurological complications or haematological complications and (iii) uteroplacental dysfunction;

3. Chronic hypertension (essential and secondary): high blood pressure predating pregnancy or recognised at $<20$ weeks of gestation; and

4. PE superimposed on chronic hypertension: chronic essential hypertension accompanied by any of the above maternal organ dysfunctions consistent with PE.

The definitions were developed according to current guidelines, such as the Canadian Hypertensive Disorders of Pregnancy Working Group, ${ }^{24}$ Society of Obstetric Medicine of Australia and New Zealand, ${ }^{25}$ American College of Obstetricians and Gynecologists ${ }^{3}$ and International Society for the Study of Hypertension in Pregnancy guidelines. $^{26}$

\section{Comparison}

Normotensive pregnant women will be used as the control group.

\section{Outcomes}

Primary outcome: asthma

Asthma is defined by a history of respiratory symptoms, including wheezing, shortness of breath, chest tightness and cough, that vary over time and in intensity in addition to variable expiratory airflow limitation according to the Global Initiative for Asthma ${ }^{12}$ and International Collaboration in Asthma, Allergy and Immunology. ${ }^{27}$

\section{Secondary outcomes}

1. Wheezing/recurrent wheezing;

a. Lung/pulmonary function (including forced expiratory volume in $1 \mathrm{~s}\left(\mathrm{FEV}_{1}\right)$, forced expiratory volume at $0.5 \mathrm{~s}$, forced vital capacity $(\mathrm{FVC}), \mathrm{FEV}_{1} /$ FVC, $50 \%$ of the forced expiratory flow and $75 \%$ of the forced expiratory flow, maximal mid-expiratory flow), active asthma, asthma exacerbations and asthma treatment (including hospitalisation, systemic corticosteroid use, inhaled $\beta_{2}$-agonist use, inhaled corticosteroid use, combination of inhaled $\beta_{2}$ agonists and corticosteroid use, leukotriene antagonist use and other anti-asthma medication use); and

2. Serum immunoglobulin E levels. 


\section{METHOD}

The systematic review and meta-analysis will follow the Preferred Reporting Items for Systematic Reviews and Meta-Analysis (PRISMA) ${ }^{28}$ and Meta-analysis of Observational Studies in Epidemiology guidelines. ${ }^{29}$

\section{Criteria for considering studies for review Inclusion criteria}

1. Studies with cohort, case-control and cross-sectional designs that report a diagnosis of HDP and asthma in offspring as the outcome of interest;

2. Studies that estimate the relationship between HDP and asthma and report the estimated risks (ORs or relative risks (RRs)) with 95\% CIs or provide sufficient information to calculate these values;

3. Studies published in only the English language;

4. Studies published in any year; and

5. Studies that include only humans.

\section{Exclusion criteria}

1. Conference abstracts, letters to the editor, reviews and commentary articles;

2. Studies with overlapping data; or

3. Studies missing raw data.

\section{Search strategy}

A systematic search of the literature will be conducted in the PubMed, Embase, Cochrane and Web of Science databases from database inception through 31 December 2019. We will search databases such as Google Scholar for grey literature. A three-phase search strategy will be applied in this review.

1. For HDP, the following combination of search terms will be used: 'hypertensive disorders during pregnancy' or 'hypertensive disorders in pregnancy' or 'hypertensive disorders of pregnancy' or 'pregnancy-induced hypertension' or 'eclampsia' or 'preeclampsia' or 'preeclampsia' or 'preeclamptic pregnancy' or 'chronic hypertension' or 'gestational hypertension' or 'chronic hypertension with superimposed preeclampsia' or 'postpartum hypertension' or 'maternal hypertensive disorders'.

2. For asthma, the following search terms will be used: 'asthma' or 'wheeze' or 'wheezing' or 'shortness of breath or 'bronchial spasm' or 'bronchospasm' or 'bronchoconstriction' or 'bronchoconstrict' or 'bronchial hyperreactivity or 'bronchial hyperresponsiveness' or 'reactive airway disease' or 'obstructive lung disease' or 'air low limitation or chronic obstructive respiratory disorder' or 'chronic obstructive pulmonary disease'.

3. We will combine steps 1 and 2 with 'AND'.

The detailed search strategy is listed in online supplementary table 1 . We will manually search the reference lists of the included studies to further identify eligible studies.

\section{Study selection and data extraction}

The eligibility of each study will be assessed independently by two investigators (PL and TX), and disagreements will be solved by discussion (with a third author, $\mathrm{YH}$, when necessary). Data from the identified studies will be extracted using the standardised data extraction form listed in online supplementary table 2. The titles and abstracts of the studies retrieved from each database will be stored and managed in the EndNote reference manager. For each included study, we will extract the following information: first author's last name, year of publication, study location, study design, sample size, ascertainment of exposure (HDP and its subgroups), outcome diagnostic criteria, offspring age at diagnosis, adjusted/matched confounding variables and effects estimates (RRs or ORs) with 95\% CIs. The eligibility of each study will be assessed independently by two investigators (PL and TX), and disagreements will be solved by discussion (with a third author, $\mathrm{YH}$, when necessary).

\section{Subgroups/subsets}

Subgroup analyses will be carried out according to the following: (1) study design (cohort vs case-control vs cross-sectional), (2) sample size, (3) location (eg, Europe vs USA), (4) income level of the country (low/middle/ high), (5) year of publication, (6) study subject selection, (7) study quality (minimal/low vs moderate/high), (8) adjusted offspring factors (eg, age at diagnosis of asthma, sex, birth weight, gestational age) and (9) adjusted maternal factors (eg, maternal asthma, maternal body mass index, gestational weight gain, maternal smoking). Sensitivity analyses will be performed by excluding each study one by one and calculating a pooled estimate for the remainder of the studies.

If any other subgroup/sensitivity analyses are identified during the process of the meta-analysis, these will be clearly labelled as post hoc analyses.

\section{Risk of bias (quality) assessment}

The quality of cohort and case-control studies will be assessed using the Newcastle-Ottawa Scale ${ }^{30}$ which evaluates the selection of study groups (one star for each term), comparability (up to two stars) and exposure or outcome (one star for each term) (online supplementary table 3). A high score indicates a low risk of bias. Additionally, cross-sectional studies will be assessed using the Agency for Healthcare Research and Quality-recommended 11 items scale (online supplementary table 4). ${ }^{31}$ Furthermore, the Grading of Recommendations, Assessment, Development and Evaluation (GRADE) methodology will be used to evaluate the overall likelihood of quality by examining six types of bias in each study (selection, exposure, outcome, analytical, attrition and confounding) (online supplementary table 5) ${ }^{32}$ The GRADE classifies the quality of studies as low, moderate and high. The quality assessment of each study will be independently carried out by two authors, and any disagreements will be resolved through discussion (with a third author when necessary). We will use funnel plots to assess publication bias, and Egger's linear regression will be applied to test for funnel plot asymmetry. 


\section{Strategy for data synthesis}

The overall pooled estimates for the association between HDP and asthma will be calculated in the meta-analysis. We will use the generic inverse variance method to determine both the crude and adjusted results. A summary OR estimate with a $95 \%$ CI will be calculated by using fixedeffect and random effects models. The $\mathrm{I}^{2}$ statistic will be applied to examine heterogeneity. According to the Cochrane Handbook criteria, if the $\mathrm{I}^{2}$ value is less than $50 \%$, heterogeneity is low, and a fixed-effect model will be used in the analysis. Otherwise, the heterogeneity will be considered high if the $\mathrm{I}^{2}$ value is $50 \%$ or more, and a random effects model will be used. Forest plots will be constructed to show the study-specific RR/OR estimates and pooled RR/OR estimates. If a study is eligible for inclusion in the systematic review but does not provide adequate data for inclusion in the meta-analysis, a narrative synthesis will be conducted to summarise and tabulate the results. All analyses will be performed in Stata 14 and RevMan 5.3.

\section{Presenting and reporting the results}

The study selection procedure will be outlined by a flow diagram according to the PRISMA statement in the metaanalysis (online supplementary figure 1 ). In addition, the reason for excluding studies will be presented. The characteristics of each included study, including the population, age range of participants, sample size, year range of studies, study design, country, exposure, outcome, measure of effect, unadjusted or adjusted effects (95\% CI) and adjustment for covariates, will be tabulated in detail. The heterogeneity of the results will be listed in another table. Additionally, we will use forest plots to present the pooled estimates in the meta-analysis. Eligible studies for which we could not obtain raw data by contacting the corresponding authors will be listed individually in a separate table.

\section{Patient and public involvement}

This systematic review and meta-analysis will be based on published studies; therefore, primary patient data will not be collected. Patients and the public will not be involved in the study design, recruitment and data analysis.

\section{Summary}

In this systematic review and meta-analysis, we will assess the association between HDP and asthma in offspring by summarising the existing literature based on a predesigned protocol. Our results might help reveal the potential aetiology of asthma involving fetal lung and immune system development during the prenatal period. Thus, the supporting evidence may promote strengthened surveillance of HDP-exposed infants, leading to early identification and intervention and improved lung function and asthma outcomes.

Acknowledgements We acknowledge suggestions from Imti Choonara (Emeritus Professor in Child Health and Editor in Chief of BMJ Paeds Open, University of
Nottingham, the Medical School, Derby, UK) on the conception and design of the analysis as well as other valuable advice regarding this manuscript.

Contributors The study was conceived by PL and TX. PL, TX and YH developed the eligibility criteria, search strategy, assessment of methodological quality, data extraction methods and data summary plan. PL and TX wrote the protocol. TX supervised the work. All authors approved the final version.

Funding This work is supported by the National Science Foundation of China (No. 81300525 to TX, No. 81701540 to PL), the Deep Underground Space Medical Center (No. DUGM201809 to TX) and Fundamental Research Funds for the Central Universities (to TX).

Competing interests None declared.

Patient and public involvement Patients and/or the public were not involved in the design, or conduct, or reporting or dissemination plans of this research.

Patient consent for publication Not required.

Provenance and peer review Not commissioned; externally peer reviewed.

Open access This is an open access article distributed in accordance with the Creative Commons Attribution Non Commercial (CC BY-NC 4.0) license, which permits others to distribute, remix, adapt, build upon this work non-commercially, and license their derivative works on different terms, provided the original work is properly cited, appropriate credit is given, any changes made indicated, and the use is non-commercial. See: http://creativecommons.org/licenses/by-nc/4.0/.

ORCID iD

Tao Xiong http://orcid.org/0000-0001-5255-6324

\section{REFERENCES}

1 Gillon TER, Pels A, von Dadelszen P, et al. Hypertensive disorders of pregnancy: a systematic review of international clinical practice guidelines. PLoS One 2014;9:e113715-22.

2 Olson-Chen C, Seligman NS. Hypertensive emergencies in pregnancy. Crit Care Clin 2016;32:29-41.

3 American College of Obstetricians and Gynecologists, Task Force on Hypertension in Pregnancy. Hypertension in pregnancy. Report of the American College of Obstetricians and Gynecologists' Task Force on Hypertension in Pregnancy. Obstet Gynecol 2013;122:1122-31.

4 Stokholm J, Sevelsted A, Anderson UD, et al. Preeclampsia associates with asthma, allergy, and eczema in childhood. Am J Respir Crit Care Med 2017;195:614-21.

5 Pinheiro TV, Brunetto S, Ramos JGL, et al. Hypertensive disorders during pregnancy and health outcomes in the offspring: a systematic review. J Dev Orig Health Dis 2016;7:391-407.

6 Boyd HA, Basit S, Behrens I, et al. Association between fetal congenital heart defects and maternal risk of hypertensive disorders of pregnancy in the same pregnancy and across pregnancies. Circulation 2017;136:39-48.

7 Zheng J-S, Liu H, Ong KK, et al. Maternal blood pressure rise during pregnancy and offspring obesity risk at 4 to 7 years old: the Jiaxing birth cohort. J Clin Endocrinol Metab 2017;102:4315-22.

8 Tuovinen S, Eriksson JG, Kajantie E, et al. Maternal hypertensive pregnancy disorders and cognitive functioning of the offspring: a systematic review. J Am Soc Hypertens 2014;8:832-47.

9 Maher GM, O'Keeffe GW, Kearney PM, et al. Association of hypertensive disorders of pregnancy with risk of neurodevelopmenta disorders in offspring: a systematic review and meta-analysis. JAMA Psychiatry 2018;75:809-19.

10 Gillman MW. Developmental origins of health and disease. N Engl J Med 2005;353:1848-50.

11 Bousquet J, Anto JM, Berkouk K, et al. Developmental determinants in non-communicable chronic diseases and ageing. Thorax 2015;70:595-7.

12 Boulet L-P, Reddel HK, Bateman E, et al. The Global Initiative for Asthma (GINA): 25 years later. Eur Respir J 2019;54:1900598.

13 Huang K, Yang T, Xu J, et al. Prevalence, risk factors, and management of asthma in China: a national cross-sectional study. Lancet 2019;394:407-18.

14 Cookson W. The alliance of genes and environment in asthma and allergy. Nature 1999;402:5-11.

15 Kleeberger SR, Peden D. Gene-Environment interactions in asthma and other respiratory diseases. Annu Rev Med 2005;56:383-400.

16 Masoli M, Fabian D, Holt S, et al. The global burden of asthma: Executive summary of the GINA dissemination Committee report. Allergy 2004;59:469-78. 
17 Bobolea I, Arismendi E, Valero A, et al. Early life origins of asthma: a review of potential effectors. J Investig Allergol Clin Immunol 2019;29:168-79.

18 Håland G, Carlsen KCL, Sandvik L, et al. Reduced lung function at birth and the risk of asthma at 10 years of age. $N$ Engl J Med 2006;355:1682-9.

19 Harding R, Maritz G. Maternal and fetal origins of lung disease in adulthood. Semin Fetal Neonatal Med 2012;17:67-72.

20 Postma DS, Bush A, van den Berge M. Risk factors and early origins of chronic obstructive pulmonary disease. Lancet 2015;385:899-909.

21 Simpson CR, Sheikh A. Trends in the epidemiology of asthma in England: a national study of 333,294 patients. J $R$ Soc Med 2010;103:98-106.

22 Liu X, Olsen J, Agerbo E, et al. Maternal preeclampsia and childhood asthma in the offspring. Pediatr Allergy Immunol 2015;26:181-5.

23 Byberg KK, Ogland B, Eide GE, et al. Birth after preeclamptic pregnancies: association with allergic sensitization and allergic rhinoconjunctivitis in late childhood; a historically matched cohort study. BMC Pediatr 2014;14:101-7.

24 Magee LA, Pels A, Helewa M, et al. Diagnosis, evaluation, and management of the hypertensive disorders of pregnancy: Executive summary. J Obstet Gynaecol Can 2014;36:416-38.

25 Lowe SA, Bowyer L, Lust K, et al. SOMANZ guidelines for the management of hypertensive disorders of pregnancy 2014. Aust N Z J Obstet Gynaecol 2015;55:e1-29.
26 Brown MA, Magee LA, Kenny LC, et al. Hypertensive disorders of pregnancy: ISSHP classification, diagnosis, and management recommendations for international practice. Hypertension 2018;72:24-43.

27 Papadopoulos NG, Arakawa H, Carlsen K-H, et al. International consensus on (icon) pediatric asthma. Allergy 2012;67:976-97.

28 Moher D, Shamseer L, Clarke M, et al. Preferred reporting items for systematic review and meta-analysis protocols (PRISMA-P) 2015 statement. Syst Rev 2015;4:1.

29 Stroup DF, Berlin JA, Morton SC, et al. Meta-Analysis of observational studies in epidemiology: a proposal for reporting. meta-analysis of observational studies in epidemiology (moose) group. JAMA 2000;283:2008-12.

30 Stang A, Andreas S. Critical evaluation of the Newcastle-Ottawa scale for the assessment of the quality of nonrandomized studies in meta-analyses. Eur J Epidemiol 2010;25:603-5.

31 Rostom A, Dube C, Cranney A, et al. Celiac disease. Rockville (MD): agency for healthcare research and quality (US); 2004 Sep. (evidence Reports/Technology assessments, no. 104.) appendix D. quality assessment forms. Available: http://www.ncbi.nlm.nih.gov/books/ NBK35156 [Accessed 2004].

32 Andrews J, Guyatt G, Oxman AD, et al. Grade guidelines: 14. going from evidence to recommendations: the significance and presentation of recommendations. J Clin Epidemiol 2013;66:719-25 